\title{
End-User Development: From Creating Technologies to Transforming Cultures
}

\author{
Gerhard Fischer \\ Center for LifeLong Learning \& Design (L3D) \\ Department of Computer Science and Institute of Cognitive Science \\ University of Colorado, Boulder USA \\ gerhard@colorado.edu
}

\begin{abstract}
In a world that is not predictable, improvisation, evolution, and innovation are more than luxuries: they are necessities. The challenge of design is not a matter of getting rid of the emergent, but rather of including it and making it an opportunity for more creative and more adequate solutions to problems. End-user development (EUD) provides the enabling conditions for putting owners of problems in charge by defining the technical and social conditions for broad participation in design activities. It addresses the challenges of fostering new mindsets, new sources of creativity, and cultural changes to create foundations for innovative societies.

Grounded in the analysis of previous research activities this paper explores (1) conceptual frameworks for EUD (including: socio-technical environments; metadesign; and cultures of participation), (2) models guiding and supporting EUD (including: the seeding, evolutionary growth, reseeding process model; ModelAuthoritative and Model-Democratic for knowledge creation, accumulation, and sharing; and richer ecologies of participation). These frameworks and models are briefly illustrated in one specific application domain.

The paper concludes by articulating design guidelines and design-tradeoffs to shape the future of EUD being understood as a cultural transformation rather than only as a technology to create software artifacts.

Keywords: socio-technical environments, meta-design, cultures of participation, seeding, evolutionary growth, reseeding process model, Model-Authoritative, Model-Democratic, ecologies of participation, personally meaningful problems, control, participation overload, future research agenda for EUD, design guidelines, design trade-offs
\end{abstract}

\section{Introduction}

In a world where change is the norm, end-user development (EUD) is a necessity rather than a luxury because it is impossible to design artifacts (including: software systems, socio-technical environments, and learning environments) at design time for all the problems that occur at use time. The co-evolution of systems and users' practices requires socio-technical environments that can evolve and be tailored continuously. Figure 1 provides an overview and illustrates the structure of this paper that tries to articulate a 
theoretically-guided and empirically-supported vision for the future of EUD as a cultural transformation rather than only as a technology to create software artifacts.

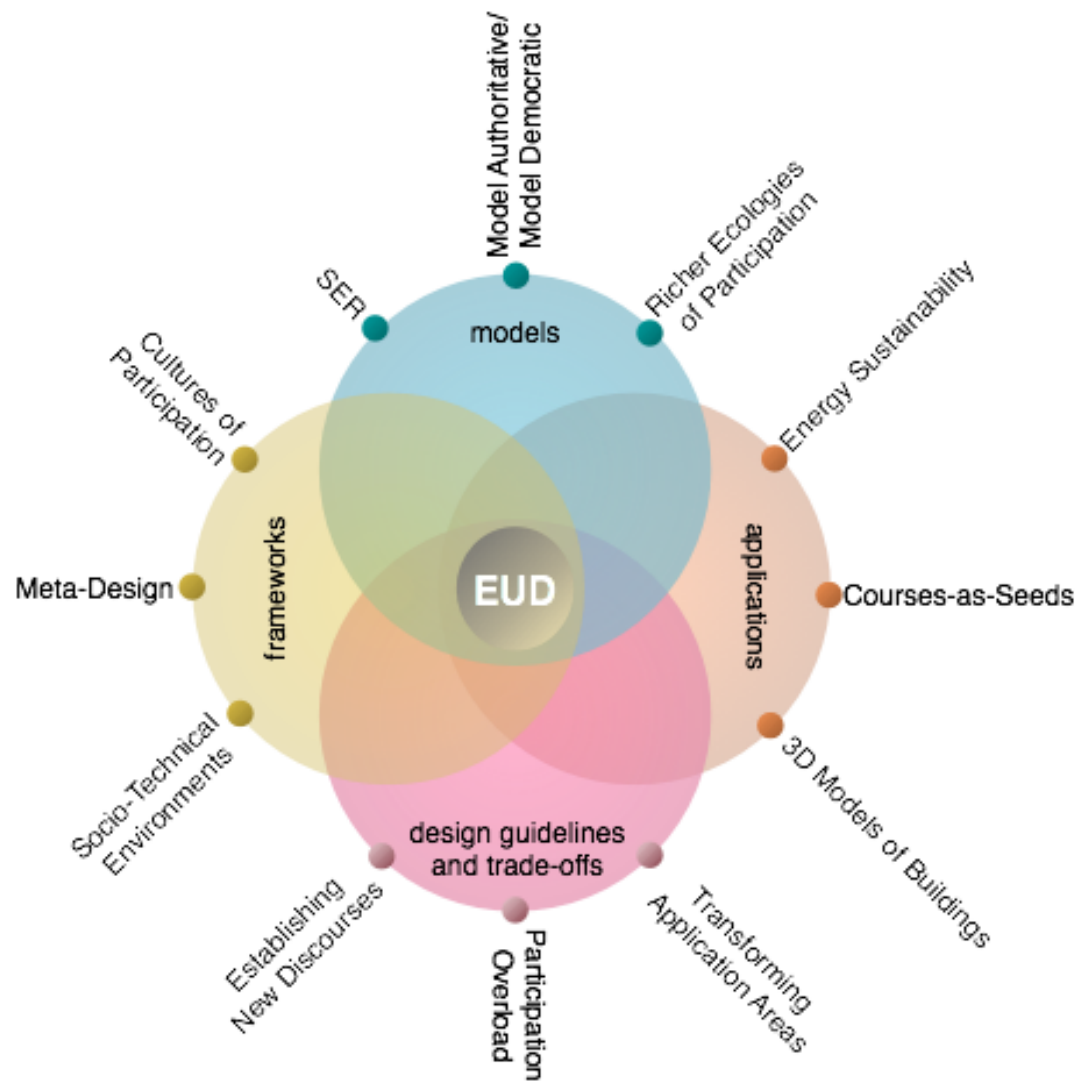

Figure 1: Conceptualizing EUD as Cultural Transformation

\section{EUD: From Creating Technologies to Transforming Cultures}

EUD is instrumental for "the ability to reformulate knowledge, to express oneself creatively and appropriately, and to produce and generate information rather than simply to comprehend it" [1]. It appeals to diverse audiences by supporting them in designing and building their own artifacts by situating computation in new contexts, by generating content, and by developing tools that democratize design, innovation, and knowledge creation [2]. This broad vision of EUD complements and transcends a technological perspective of EUD [3] that is closely related to:

- End-User Programming (EUP) that empowers and supports end-users to program (with techniques such as: programming by demonstration, visual programming, scripting languages, and domain-specific languages) [4]; and 
- End-User Software Engineering (EUSE) that adds to EUP support for systematic and disciplined activities for the whole software lifecycle (including: reliability, efficiency, usability, debugging support, and version control) [5].

Addressing Important Problems. A cultural transformation perspective of EUD includes technological developments as essential components but transcends them with additional objectives addressing requirements derived from the following fundamental problems [6]:

- problems of a magnitude which individuals and even large teams cannot solve and require the contribution of all interested citizens - illustrated by examples such as (1) to model all buildings in the world in 3-D addressed by "SketchUp and 3D Warehouse" and (2) to cope with the immense amount of local knowledge needed for "Google Map Maker");

- problems of a systemic nature requiring the collaboration of many different minds from a variety of backgrounds - illustrated by the growing importance of application domain knowledge for most software systems and the fact that this knowledge is held by end-users and domain experts rather than by software developers who suffer from a "thin spread of application domain knowledge" [7];

- problems modeling changing and unique worlds supported by open and evolvable systems based on fluctuating and conflicting requirements illustrated by the emerging mismatches between evolving worlds and the systems which model these world [8]).

Inspirations, Perspectives and Influences for Conceptualizing EUD as a Transformational Culture. An early inspiration for conceptualizing EUD as a transformational culture was articulated by Ivan Illich with convivial systems envisioned to "give each person who uses them the greatest opportunity to enrich the environment with the fruits of his or her vision" [9]. To cope with the unattainable challenge of fully anticipating or envisioning use before actual use takes place in people's life worlds, participatory design ("design for use before use") was complemented with meta-design ("design for design after design") [10]. An interesting perspective on EUD are nudges defined by choice architects which try to motivate people to engage in certain actions and behavior, but provide them with EUD opportunities to have complete choice over their own actions by supporting a methodology characterized as "libertarian paternalism" [11]. A current development relying on EUD is technology mediated social participation, a movement that pursues empowering individuals to become active in local and global communities with a focus on exploring questions of how to motivate participation, increase social trust, and promote collaboration [12].

Related Research Efforts. The conceptualization of EUD as a transformational culture has been explored by a number of research activities including:

- the Software Shaping Workshops environment [13] allows end users to carry out their activities and adapt environment and tools without the burden of using a traditional programming language, but using high-level visual languages tailored to their needs. It has been explored in various interactive software systems including the medical domain, the mechanical engineering domain, and the tourism and cultural heritage domain; 
- the hive-mind space (HMS) model [14] supports collaborative design activities of multidisciplinary design teams and it integrates and evolve the software shaping workshop methodology and the seeding, evolutionary growth, reseeding (SER) process model with a focus on fostering creativity;

- meta-design based theories have been applied and analyzed in virtual worlds (specifically massively-multiplayer online role-playing games such as 'Lord of the Rings Online' and open-ended virtual worlds such as Second Life) [15] with the objective to increase their EUD potential with insights from different domain;

- the impact of different relationship between design and use on EUD has been studied by [16] with the objective to identify the most promising approaches to shift design power to the users.

\section{Conceptual Frameworks for Exploring EUD as a Transformational Culture}

Our research over the last decade has articulated and assessed different conceptual frameworks that provide foundations to explore, foster, and support EUD as a transformational culture.

Socio-Technical Environments. Socio-technical environments [17] are focused on the systematic integration of two sets of design requirements: (1) technical components (computers, networks, building materials, and software substrates) and (2) social components (people, procedures, laws, collaboration and communication policies). The impact of socio-technical environments on EUD [18] has explored the following dimensions: (1) support for cultures of participation that put the owners of problems in charge and give them control of how technical systems are used and which functionality is underlying the usage; (2) mechanisms to support empowerment for adaptation and evolution at use time by offering functionality for tailorability, customization, and userdriven adaptability; (3) system developments based on the seeding, evolutionary growth, and reseeding model, in which the seed represents basic structures and is in accordance with the relevant standards but it leaves space and options for the development of concrete details; (4) semi-structured modeling to support and accompany the communication during the evolution of a socio-technical system by documenting and providing specification of details for requirements, plans, technical specifications, and business processes; and (5) walkthrough-oriented facilitation to support the integration of various perspectives, the negotiation of design decisions, and the building of commitments about how technology will be used and adapted, and the evaluation of prototypes.

Meta-design. Meta-design is "design for designers" [19]. It represents an emerging conceptual framework aimed at defining and creating social and technical infrastructures in which participatory cultures can come alive and new forms of collaborative design can take place. It extends the traditional notion of system design beyond the original development of a system to allow users become co-designers and co-developers [20]. It is grounded in the basic assumption that future uses and problems cannot be completely anticipated at design time, when a system is developed. Users, at use time, will discover 
mismatches between their needs and the support that an existing system can provide for them. These mismatches will lead to breakdowns that serve as potential sources of new insights, new knowledge, and new understanding. Meta-design addresses the following features of socio-technical environments: (1) it allows them to be flexible and evolve because they cannot be completely designed prior to use; (2) the end-users are the primary agents to drive the evolution; and (3) the meta-designers design systems explicitly for evolution.

Cultures of Participation. The rise in social computing (based on social production and mass collaboration) has facilitated a shift from consumer cultures (specialized in producing finished artifacts to be consumed passively) to cultures of participation (in which all people are provided with the means to participate and to contribute actively in personally meaningful problems) $[6,21]$. These developments represent unique and fundamental opportunities, challenges, and transformative changes for EUD as we move away from a world in which a small number of people define rules, create artifacts, make decisions for many consumers towards a world in which everyone has possibilities to actively participate by creating widely accessible artifacts. These transformations will create foundations for a different kind of society than one where citizens, workers and learners are confined to the role of consumers [22].

Cultures of participation are facilitated and supported by a variety of different technological environments (such as: the participatory Web ("Web 2.0"), table-top computing, domain-oriented design environments); all of them contributing in different ways to the aims of engaging diverse audiences, enhancing creativity, sharing information, and fostering the collaboration among users acting as active contributors and designers. They democratize design and innovation [2] by shifting power and control towards users, supporting them to act as both designers and consumers ("prosumers" [23]) and allowing systems to be shaped through real-time use.

\section{Models Guiding and Supporting EUD}

An important objective for the EUD perspective articulated in this article is that design as a process is tightly coupled to use and it continues during the use of the system [20]. It sees the "unfinished" as an opportunity (by extending design time indefinitely) rather than as an obstacle or as something to be avoided. The models briefly described in this section address three different aspects: (1) designing seeds (that can grow) rather than complete systems; (2) democratizing design by replacing "filter then publish" with "publish then filter" approaches; and (3) creating richer ecologies of participation.

The Seeding, Evolutionary Growth, Reseeding (SER) Process Model. The SER model [8] is a descriptive and prescriptive model for creating the social and technical infrastructures in which new forms of collaborative design can take place that best fit an emerging and evolving context. Instead of attempting to build complete systems, the SER model advocates building seeds that can evolve over time. It postulates that systems that evolve over a sustained time span must alternate between (1) periods of planned activity and unplanned evolution, and (2) periods of deliberate (re)structuring and enhancement. A seed is something that has the potential to change and grow. In socio-technical 
environments, seeds need to be designed and created for the technical as well as the social component of the environment.

A seed is built based on an initial understanding and framing of the problem. It is created by meta-designers [19] and choice architects [11]) for future users to be as complete as possible. However, the designed system cannot be truly complete due to the situated and tacit nature of knowledge work. Furthermore, the constant changes occurring in the environment in which the system is embedded will breed new needs, and the introduction of a computational system itself generates changes in professional practices and sociotechnical environments. Therefore, the initial seed needs to be adapted to the new understanding and new environments.

The evolutionary growth phase is one of decentralized evolution as the seed is used and extended by users to do their work or explore new problems. In this phase, meta-designers are not directly involved because the focus has shifted to problem framing and problem solving activities of users who have a direct stake in the problem at hand.

During the evolutionary growth phase, the seed plays two roles simultaneously: (1) it provides resources for work (solutions that have been accumulated from prior use), and (2) it accumulates the products of work, as each project may contribute new solutions and extension to the seed. During the evolutionary growth phase, users focus on solving specific problems and creating problem-specific solutions rather than on creating general solutions. As a result, the solutions added during this phase may not be well integrated with the rest of the solution in the seed.

Reseeding is a deliberate and centralized effort to organize, formalize, and generalize solutions and artifacts created during the evolutionary growth phase. The goal of reseeding is to create an information space in which useful solutions can be easily found, reused, and extended. As in the seeding phase, professional software developers are needed to perform substantial system and solution space modifications, but users must participate because only they can judge what solutions are useful and what structures will serve their work practices.

MODEL-AuthORITATIVE and Model-Democratic: Re-conceptualizing Knowledge Creation, Accumulation, and Sharing. Knowledge creation, accumulation, and sharing in society has undergone major changes as illustrated by contrasting different models for these processes.

ModeL-AUthoRItative. Professionally dominated design cultures (see Figure 2) supported by broadcast technologies are characterized by a small number of experts acting as contributors and a large number of passive consumers. In such cultures, strong input filters exist based on:

- substantial knowledge is necessary for contributions (e.g.: the in-depth understanding of established fields of inquiry or the need to learn specialized high-functionality tools);

- extensive quality control mechanisms exist (e.g.: the certification of professionals or low acceptance rates for conference and journal articles); and

- large organizations and high investments for production are required (e.g.: film studios such as Hollywood, newspaper production facilities). 


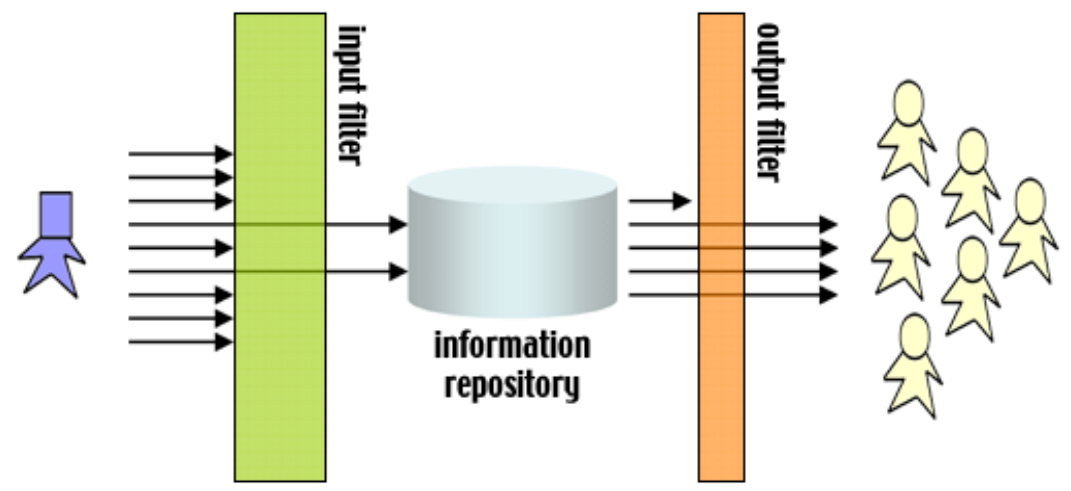

Figure 2: Model-Authoritative underlying Professionally Dominated Cultures

A consequence of the strong input filters preventing and rejecting contributions is that relatively small information repositories are created.

The advantage of this model (this is at least the basic underlying assumption) is the likelihood that the quality and trustworthiness of the accumulated information is high because the strong input filters will reject unreliable and untrustworthy information. Based on the smaller size of the resulting information repositories, relatively weak output filters are required.

The disadvantage of this model is that it greatly limits that "all voices can be heard". Their intake is limited because with only a small number of contributors too many views are unexplored and underrepresented because the controlling mechanisms behind the input filters suppress broad participation from different constituencies. Relevant information and divergent opinions (which may be of great value not at a global level but for the work of specific individuals) will often not be included in the information repository. Most people are limited to accessing existing information, denying them a voice even in the context of personally meaningful problems and in situations in which specialized idiosyncratic knowledge would represent a unique contribution.

MODEL-DEMOCRATIC. Democratized design cultures [2, 24]) (see Figure 3) supported by Web 2.0 technologies [25] can be characterized by weak input filters allowing users not only to access information but to become active contributors by engaging in informed participation. The weak input filters result in much larger information repositories (with information repositories such as the World Wide Web being the prime example). 

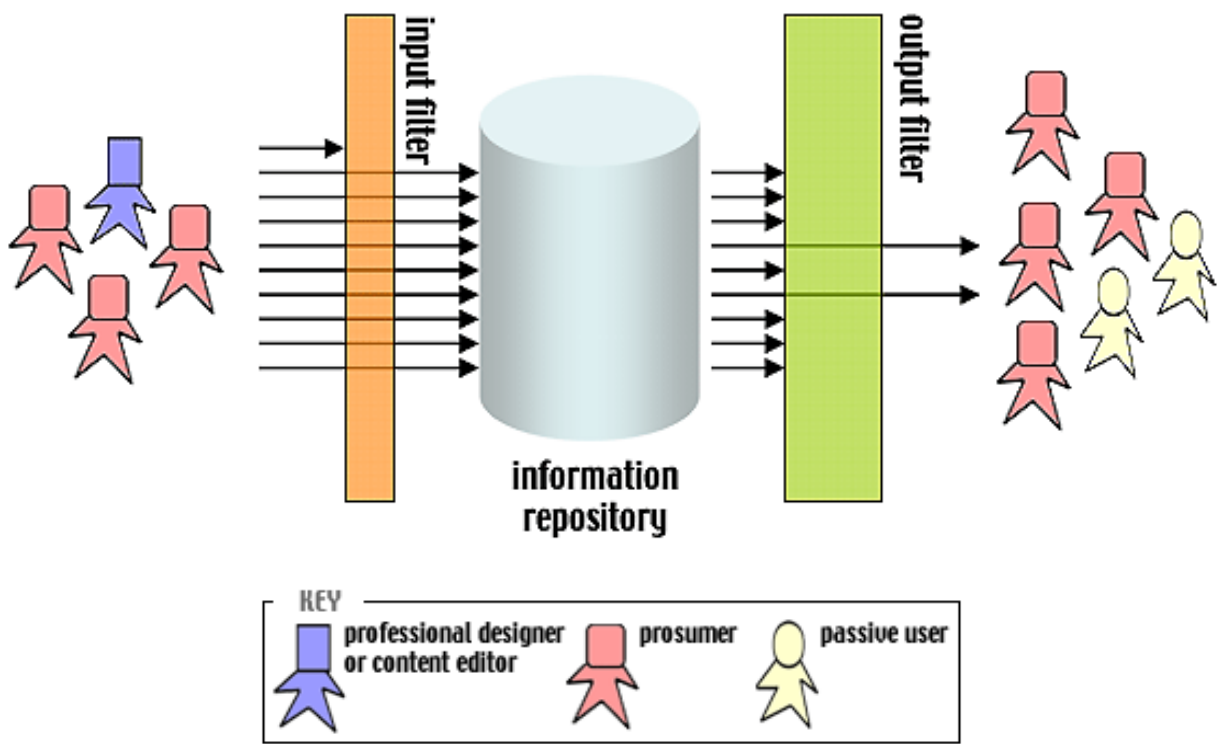

Figure 3: Model-Democratic underlying Democratic Design Cultures

MODEL-DEMOCRATIC on the technical side requires powerful tools for creating content (such as Wiki substrates and domain-oriented design environments), for organizing content (such as supporting collections), and for distributing content (such as powerful search capabilities and recommender systems). On the social side, it requires active contributors (who master the design tools and who are motivated to contribute), curators (who organize large information repositories) and docents (who assist in helping learners to identify and locate relevant information).

The advantages and disadvantages of the two models are to some extent reversed. Major limitations of MODEL-DEMOCRATIC are the potentially reduced trust and reliability of the content of the information repositories based on the weak input filter. The amount of available information is exploding, and since too much information consumes the true scarce resource of human attention, the large information repositories are a mixed blessing unless we are able to develop strong new output filters (e.g.: powerful search mechanisms to find relevant information, collaborative filtering, recommender and tagging systems, and user and task models to personalize information).

Richer Ecologies of Participation: Breaking Down the Strict Designer-User Distinction. One of the major aspects of EUD as a transformational culture is to break down the strict separation between "designer" and "user" by supporting richer ecologies of participation. Different empirical analyses have explored ecologies of participation in different domains. Our systematic analysis of several open source software systems and communities [26] has revealed a variety of roles that people take: (1) passive users; (2) readers; (3) bug reporters; (4) bug fixers; (5) peripheral developers; (6) active developers; and (7) project leaders. In our framework for cultures of participation, we have explored 
five distinct roles: (1) unaware consumers, (2) aware consumers, (3) collaborators, (4) designers, and (5) meta-designers [19, 27]).

For cultures of participation to become viable and be successful, it is critical that a sufficient number of participants take on the more active and more demanding roles [28, 29]. EUD research needs to analyze the necessary requirements associated with the more active roles, and develop social and technical interventions to support participants in their migration paths by reducing barriers to involvement and inclusion. Richer ecologies of participation can be specifically observed in communities [30] in which "gardeners, power users, and local developers" emerge who helps other users to address their needs.

\section{Application Domain: “Courses-as Seeds"}

The conceptual frameworks and models articulated in the previous sections have been explored (1) in a large number of major applications (including: open source software, Wikipedia, YouTube, Instructables, etc.), (2) by other members of the EUD research community (see section "Related Research Efforts" above), and (3) in our own work [6, 31]. This section illustrates the cultural transformation perspective of EUD in one of our own activities: teaching courses at a university.

Providing learners of all ages with the means to become co-creators of new ideas, knowledge, and products in personally meaningful activities presents one of the most exciting innovations and transformations in education with profound implications in the years to come.

Courses-as-seeds [32] is an educational approach (based on the SER model and MODELDEMOCRATIC) that explores EUD in the context of fundamentally changing the nature of courses taught in universities. It complements the currently increasingly popular approach of Massive Open Online Courses (MOOCs; based on Model-AuthORITATIVE [33]) with their promise and hype that online learning will give millions of students access to the world's best teachers.

The goals of courses-as-seeds are:

- to overcome the impoverished conception that a course provides a learning experience in which an all-knowing teacher tells or shows unknowing learners something they presumably know nothing about [34];

- to foster cultures of participation [6] by providing all students with the opportunity to contribute;

- to involve students as active contributors, because courses should not only rely on the intellectual capital provided by an instructor. Our courses (a large number of them being available at: http://13d.cs.colorado.edu/ gerhard/courses/) are conceptualized based on the seeding, evolutionary growth, reseeding model, in which the instructor provides the initial seed rather than a finished product and the content of a course evolves over time through contributions of the students; and

- to reinvent classrooms as communities of mutual learners, in which the roles of teachers and learners are not assigned to specific persons, but to specific contexts 
and in which teachers act as "guides on the side" rather than as "sages on the stage".

\section{Design Guidelines and Design Trade-Offs for EUD from a Transformational Culture Perspective}

This section briefly describes design guidelines and design trade-offs derived from the frameworks and models articulated earlier in the paper considered relevant in supporting a transformational culture perspective for future EUD developments.

\subsection{Design Guidelines}

EUD: Transforming Application Areas. Conceptualizing EUD as a transformational culture has the potential to deepen our understanding of EUD in a number of application areas including: (1) software design, with a focus on customization, personalization, tailorability [35]; and end-user programming [4]; (2) teaching and learning, with a focus on learning communities and courses-as-seeds [32]; and (3) information repositories (based on Web 2.0-based technologies) [25]

Supporting and Fostering Cultures of Participation. EUD supports cultures of participation by offering and supporting possibilities for making changes. Contributors should not be intimidated and should not have the impression that they are incapable of making changes; the more users become convinced that changes are not as difficult as they think they are, the more they may be willing to participate. Beyond making changes possible, benefits must be perceived by the contributors who must believe that what they get in return justifies the investment they make. The benefits perceived may vary and can include: professional benefits (helping for one's own work), social benefits (increased status in a community, possibilities for jobs), and personal benefits (engaging in fun activities). To reap the benefits of individual contributions by the whole community, low barriers and rewards must exist for sharing changes.

Defining the Role of Meta-Designers. Meta-designers need to use their own creativity to develop socio-technical environments in which other people can be creative. They must create the social conditions for broad participation in design activities being as important as creating artifacts. The main activity of meta-designers shifts from determining the meaning, functionality, and content of a system to define contexts that encourage and support users to participate. Meta-designers must be willing to share control of how systems will be used, which content will be contained, and which functionality will be supported.

Harness Social Creativity. Meta-design and cultures of participation challenge the assumption that information must move from professional designers to passive consumers. As long as only experts (including: teachers, professionals in different disciplines, commercial producers of software and movies) can determine what is right and worthwhile to be published (as postulated by MODEL-AUTHORITATIVE), societies will never be in a position to harness people's creativity and local knowledge [2]. 
EUD: Establishing New Discourses. EUD can and should establishing new discourses, including an exploration of the following concepts:

- Motivation: Human beings are diversely motivated beings acting not only for material gain, but for psychological well-being, social integration, connectedness, social capital, recognition, and for improving their standing in a reputation economy. The motivation for going the extra step to engage in cultures of participation is based on the overwhelming evidence of the IKEA effect [36] that people are more likely to like a solution if they have been involved in its generation; even though it might not make sense otherwise. Creating something personal (such as hand-knitted sweaters and socks, home-cooked meals) even of moderate quality, has a different kind of appeal than consuming something of possible higher quality made by others.

- Control: EUD supports users as active contributors who can transcend the functionality and content of existing systems. By facilitating these possibilities, control is distributed among all stakeholders in the design process. EUD erodes monopoly positions held by professions, educational institutions, experts, and high-tech scribes [24]. Empirical evidence gathered in the context of the different design activities [36] indicates that EUD is less successful when users are brought into the process late (thereby denying them ownership) and when they are "misused" to fix problems and to address weaknesses of systems that the developers did not fix themselves.

- Changing Human Behavior: Technology alone does not determine social structure nor does it change human behavior: it creates feasibility spaces for new social practices [37] and it can persuade and motivate changes at the individual, group, and community level. EUD can change people's lives (1) by making it easier for people to do things, (2) by allowing people to explore cause-and-effect relationships, and (3) by providing value that cannot be accounted for in monetary terms. Research in behavioral psychology [36] has shown that providing feedback, goal setting, and tailored information are useful in motivating people to change their behavior.

\subsection{Design Trade-Offs}

Division of Labor versus Empowerment of Individuals. Democratizing design by putting owners of problems in charge does not mean that there is no place for professionals in the future. By arguing for the independence of owners of problems from high-tech scribes, a legitimate question to ask is whether this will reverse the division of labor that has been a major driving force in advancing our societies [38]. Professional designers play an important role in our society: most persons are not able to and do not want to build their own houses, design their own cars, or write their own software systems or sorting routines. People do not have the time to participate equally in all aspects of human life in order to become fully engaged and informed, and therefore they rely on intermediaries to act in their interests. 
The Trade-Offs between Model-AUthoritative and Model-DEMOCRATIC. The advantages and disadvantages of these two models are complementary. MODELAUTHORITATIVE often limits that "all voices can be heard" thereby excluding relevant information and divergent opinions. Most people (in environments based on this model) are limited to accessing existing information, denying them a voice in the context of personally meaningful problems and in situations in which specialized idiosyncratic knowledge would represent a unique contribution. MODEL-DEMOCRATIC leads to information overload based on the accumulation of large number of potentially irrelevant contributions.

Participation Overload and Personally Meaningful Problems. Information overload has been discussed as a fundamental problem for the information society. Participation overload will be one of the most serious problems for EUD societies. Two pitfalls should be avoided: individuals (1) should not be forced to act as active contributors in situations where they want to be consumers (this is mostly the case in the context of problems and activities which are irrelevant to people); and (2) should not be restricted to consumers where they want to be active contributors and decision makers (this is mostly the case in personally meaningful situations). While there may be a cognitive surplus [22], many people feel guilty about not participating enough. Many people expiring a lack of time and being overcommitted creates the fundamental challenge to develop criteria and to be judicious about one's participation and how to develop a culture that respects a person's choices about when and how to participate.

\section{Conclusions}

EUD has moved from nonexistent to center stage (supported by the frameworks and models discussed in this paper), and the Internet (supporting MODEL-DEMOCRATIC) has provided a socio-technical environment not stifled by a small number of elitist gatekeepers (as it is the case in many environments supported by MODEL-AUTHORITATIVE). EUD perceived as a cultural transformation will create new social realities: public and private media will co-exist and blend together and professional and amateur contributions will complement each other,

Providing all citizens with the means to become co-creators of new ideas, knowledge, and products in personally meaningful activities presents one of the most exciting innovations and transformations with profound implications in the years to come. This objective characterizes the vision behind EUD as a cultural transformation. To make this vision a reality, the EUD research community needs to establish new discourses and shared languages about concepts, assumptions, values, stories, metaphors, design approaches, and learning theories. End-users by claiming ownership in personally meaningful problems should be empowered to design, build, and evolve their own artifacts and metadesigners should create environments to foster cultures of participation. These objectives will support all citizens to situate computation in new cultural and material contexts, with socio-technical environments that democratize design. 


\section{Acknowledgements}

The author thanks the members of the Center for LifeLong Learning \& Design who have made major contributions to the frameworks, models, and systems described in this paper. The authors thanks Daniela Fogli, Monica Maceli, Julie Zhu, David Diez, Ben Koehne, Stefano Valtolina, and Tony Piccino who provided insightful comments and suggestions to an earlier version of this paper.

The research was supported in part by the following grants from the National Science Foundation: (1) "Social Creativity and Meta-Design in Lifelong Learning Communities"; (2) "A Meta-Design Framework for Participative Software Systems"; (3) "A New Generation Wiki for Supporting a Research Community in Creativity and IT"; (4) "Transformative Models of Learning and Discovery in Cultures of Participation"; and (5) "Theoretical Frameworks and Socio-Technical Systems for Fostering Smart Communities in Smart Grid Environments".

The writing of this article was facilitated by the support of a "Chair of Excellence" fellowship granted to the author by the University Carlos III of Madrid.

\section{References}

[1] National-Research-Council, Being Fluent with Information Technology. Washington, DC: National Academy Press, 1999.

[2] E. von Hippel, Democratizing Innovation. Cambridge, MA: MIT Press, 2005.

[3] M. M. Burnett and C. Scaffidi, "End-User Development," in The Encyclopedia of Human-Computer Interaction (2nd Ed), M. Soegaard and R. F. Dam, Eds., Aarhus, Denmark: The Interaction Design Foundation, 2013.

[4] H. Lieberman, et al., Eds., End User Development Dordrecht, The Netherlands: Kluwer Publishers, 2006.

[5] M. Burnett, "What is End-User Software Engineering and Why Does It Matter?," in End-User Development, V. Pipek, et al., Eds., Heidelberg: Springer, 2009, pp. 15-28.

[6] G. Fischer, "Understanding, Fostering, and Supporting Cultures of Participation," ACM Interactions vol. XVIII.3 (May + June 2011), pp. 42-53, 2011.

[7] B. Curtis, et al., "A Field Study of the Software Design Process for Large Systems," Communications of the ACM, vol. 31, pp. 1268-1287, 1988.

[8] G. Fischer, et al., "Seeding, Evolutionary Growth and Reseeding: The Incremental Development of Collaborative Design Environments," in Coordination Theory and Collaboration Technology, G. M. Olson, et al., Eds., Mahwah, NJ: Lawrence Erlbaum Associates, 2001, pp. 447-472.

[9] I. Illich, Tools for Conviviality. New York: Harper and Row, 1973.

[10] T. Binder, et al., Design Things. Cambridge, MA: MIT Press, 2011.

[11] R. H. Thaler and C. R. Sunstein, Nudge - Improving Decisions about Health, Wealth, an Happiness. London: Penguin Books, 2009. 
[12] B. Shneiderman, "National Initiative for Social Participation," Science vol. 323, pp. 1426-1427, 2009.

[13] M. F. Costabile, et al., " End User Development: The Software Shaping Workshop Approach," in End User Development, H. Lieberman, et al., Eds., Dordrecht, The Netherlands: Springer, 2006, pp. 183-205.

[14] L. Zhu, "Hive-Mind Space: A Meta-design Approach for Cultivating and Supporting Collaborative Design," PhD, Dipartimento di Informatica e Comunicazione, Università degli Studi di Milano, Milano, 2012.

[15] B. Koehne, et al., "Extending the Meta-design Theory: Engaging Participants as Active Contributors in Virtual Worlds," in End-User Development (Third International Symposium, Torre Canne, Italy, June). vol. LNCS 6654, M. F. Costabile, et al., Eds., Heidelberg: Springer, 2011, pp. 264-269.

[16] M. G. Maceli, "From Human Factors to Human Actors to Human Crafters: A Meta-Design Inspired Participatory Framework for Designing in Use," Ph.D. Dissertation, Drexel University, 2012.

[17] E. Mumford, "A Socio-technical Approach to Systems Design," Requirements Engineering, Springer, vol. 5, pp. 125-133, 2000.

[18] G. Fischer and T. Herrmann, "Socio-Technical Systems: A Meta-Design Perspective," International Journal of Sociotechnology and Knowledge Development, vol. 3, pp. 1-33, 2011.

[19] G. Fischer and E. Giaccardi, "Meta-Design: A Framework for the Future of End User Development," in End User Development, H. Lieberman, et al., Eds., Dordrecht, The Netherlands: Kluwer Academic Publishers, 2006, pp. 427-457.

[20] A. Henderson and M. Kyng, "There's No Place Like Home: Continuing Design in Use," in Design at Work: Cooperative Design of Computer Systems, J. Greenbaum and M. Kyng, Eds., Hillsdale, NJ: Lawrence Erlbaum Associates, Inc., 1991, pp. 219-240.

[21] H. Jenkins, Confronting the Challenges of Participatory Cultures: Media Education for the 21st Century. Cambridge, MA: MIT Press, 2009.

[22] C. Shirky, Cognitive Surplus - Creativity and Generosity in a Connected Age. New York, N.Y.: Penguin Press, 2010.

[23] D. Tapscott and A. D. Williams, Wikinomics: How Mass Collaboration Changes Everything. New York, NY: Portofolio, Penguin Group, 2006.

[24] G. Fischer. (2002). Beyond 'Couch Potatoes': From Consumers to Designers and Active Contributors, in FirstMonday (Peer-Reviewed Journal on the Internet). Available: http://firstmonday.org/issues/issue7_12/fischer/

[25] T. O'Reilly. (2005). What Is Web 2.0 - Design Patterns and Business Models for the Next Generation of Software. Available: http://www.oreillynet.com/pub/a/oreilly/tim/news/2005/09/30/what-is-web$\underline{20 . h t m l}$

[26] G. Fischer, et al., "The Ecology of Participants in Co-Evolving Socio-Technical Environments," in Engineering Interactive Systems (Proceedings of 2nd 
Conference on Human-Centered Software Engineering). vol. LNCS 5247, P. Forbrig, Paternò, F., Ed., Heidelberg: Springer, 2008, pp. 279-286.

[27] J. Preece and B. Shneiderman, "The Reader-to-Leader Framework: Motivating Technology-Mediated Social Participation," AIS Transactions on HumanComputer Interaction, vol. 1, pp. 13-32, 2009.

[28] A. Kittur, et al., "Power of the few vs. wisdom of the crowd: Wikipedia and the rise of the bourgeoisie," in Alt.CHI at CHI 2007; San Jose, CA., 2007.

[29] J. Porter, Designing for the Social Web. Berkeley, CA: New Riders., 2008.

[30] B. A. Nardi, A Small Matter of Programming. Cambridge, MA: The MIT Press, 1993.

[31] G. Fischer, "End-User Development and Meta-Design: Foundations for Cultures of Participation," Journal of Organizational and End User Computing vol. 22, pp. 52-82, 2010.

[32] R. dePaula, et al., "Courses as Seeds: Expectations and Realities," in Proceedings of The European Conference on Computer-Supported Collaborative Learning, P. Dillenbourg, et al., Eds., Maastricht, Netherlands, 2001, pp. 494501.

[33] P. Hyman, "In the Year of Disruptive Education," CACM, vol. 55, pp. 20-22, 2012.

[34] J. Bruner, The Culture of Education. Cambridge, MA: Harvard University Press, 1996.

[35] A. Morch, "Three Levels of End-User Tailoring: Customization, Integration, and Extension," in Computers and Design in Context, M. Kyng and L. Mathiassen, Eds., Cambridge, MA: MIT Press, 1997, pp. 51-76.

[36] D. Ariely, The Upside of Irrationality - The Unexpected Benefits of Defying Logic at Work and at Home. New York, N.Y.: HarperCollins, 2010.

[37] Y. Benkler, The Wealth of Networks: How Social Production Transforms Markets and Freedom. New Haven: Yale University Press, 2006.

[38] J. S. Brown and P. Duguid, The Social Life of Information. Boston, MA: Harvard Business School Press, 2000. 